Check for updates

Cite this: RSC Adv., 2018, 8, 12556

Received 3rd February 2018

Accepted 26th March 2018

DOI: $10.1039 / \mathrm{c} 8 \mathrm{ra01085d}$

rsc.li/rsc-advances

\section{Green synthesis of amphiphilic carbon dots from organic solvents: application in fluorescent polymer composites and bio-imaging $\dagger$}

\author{
Jiangjiang Gu, (D) a Xinle Li, ${ }^{\text {b }}$ Donghua Hu, ${ }^{a}$ Yanfeng Liu, ${ }^{a}$ Guiyang Zhang, ${ }^{a}$ \\ Xudong Jia, ${ }^{a}$ Wenyu Huang (D)*b and Kai Xi (D)*a
}

\begin{abstract}
Carbon dots (CDs) have sparked tremendous attention due to their unique properties and vast potential in diverse fields. Herein, we report a green and cost-effective hydrothermal route for the synthesis of a series of CDs from readily available organics solvents. Since the organics were completely recyclable after the separation of CDs, this method holds immense potential for the large-scale synthesis of CDs. We found the DMF-CDs and DMAc-CDs possessed amphiphilicity and the diameter of amphiphilic DMF-CDs was ca. $3.5 \mathrm{~nm}$ with a narrow distribution. Moreover, these amphiphilic CDs emitted blue light under UV irradiation $(365 \mathrm{~nm}$ ) and the quantum yield could reach more than $30 \%$. Due to their good solubility in organic solvent, DMF-CDs were successfully imbedded into polymers (i.e., PS and PMMA), which revealed their potential in painting, coating, and optical devices. In addition, benefiting from high quantum yield and low cytotoxicity, the DMF-CDs in aqueous media were used as fluorescent probes in living cells, which demonstrated their great potential in bio-imaging.
\end{abstract}

\section{Introduction}

Carbon dots (CDs) or carbon quantum dots, with a typical diameter below $10 \mathrm{~nm}$, have emerged as potent metal-free fluorescent nanomaterials in the past decade. Due to their electronic transfer property, ${ }^{1}$ bandgap transitions, ${ }^{2}$ and surface defects, ${ }^{3}$ CDs exhibit an emission dependence on the excitation wavelength or surface passivation. ${ }^{4}$ Compared to the conventional semiconductor quantum dots, ${ }^{5}$ CDs possess the distinctive advantages of chem high biocompatibility, ${ }^{6}$ good watersolubility, low cytotoxicity, ${ }^{7}$ functional diversity, ${ }^{8}$ convenient synthesis, and low cost. ${ }^{9} \mathrm{~A}$ great deal of research endeavors have been devoted to the synthesis of CDs. A myriad of top-down approaches have been developed for the synthesis of CDs. However, these methods typically require toxic chemical reagents and special equipment (e.g., microwave irradiation, ${ }^{\mathbf{1 0}}$ laser ablation, ${ }^{11}$ etc.) in the exfoliation process. Instead, bottomup approaches, such as sovlothermal carbonization of glucose, chitosan, glycol, and citric acids, have spurred significant attention recently. ${ }^{12}$ Despite significant advances have been made in this area, it is of continuous and considerable interest

${ }^{a}$ Department of Polymer Science \& Engineering, Nanjing University, Nanjing 210093, PR China.E-mail:xikai@nju.edu.cn

${ }^{b}$ Department of Chemistry, Iowa State University, Ames, Iowa 50011, USA. E-mail: whuang@iastate.edu

$\dagger$ Electronic supplementary information (ESI) available. See DOI: $10.1039 / \mathrm{c} 8 \mathrm{ra} 01085 \mathrm{~d}$ to develop fluorescent CDs in an efficient, facile and green manner.

On the other hand, CDs have been widely exploited in biological applications such as cell imaging, ${ }^{13}$ biosensors, ${ }^{14}$ ions testing, ${ }^{15}$ and drug delivery, ${ }^{16}$ because of their good biocompatibility, robust chemical stability, and high resistance to photobleaching. In these applications CDs were designed to be soluble in aqueous media.

Besides aforementioned biological applications by watersoluble CDs, amphiphilic CDs, which receive relative less attention, could open up new applications in hydrophobic environments as well. For example, considering the excellent photoluminescence, low cost and biocompatibility, CDs are excellent alternative of current organic printing dyes in printing industries. However, the current methods for the synthesis of amphiphilic CDs are always associated with low yield and high cost, which severely restrains their further utilization in printing, fluorescent composites synthesis and biology related applications.

In this context, we report the synthesis of a series of CDs through a facile, one-step hydrothermal treatment of $N, N^{\prime}$ dimethylformamide (DMF), $N, N^{\prime}$-dimethylacetamide (DMAc) and other organic solvents. With this approach, amphiphilic DMF-CDs were achieved with simultaneous surface passivation and tunable fluorescence emission. This synthetic route offers multiple advantages. First, it represents a rare example of simple synthesis of amphiphilic CDs from the readily available organic solvents for the first time, which is an appealing approach from the perspective of energy and cost. Remarkably, 
the organic solvents were completely recyclable after the separation of CDs and by-products were not observed, which could achieve high atomic efficiency, one of the core focuses in green chemistry. Last, the surface passivation of DMF-CDs took place simultaneously in the hydrothermal process, rendering intrinsic fluorescence emission. Due to the good solubility in polar organic solvents (i.e., DMF), the as-synthesized DMF-CDs could be easily doped into polymer materials and the resultant CDs-polymers composites exhibit high fluorescent properties. Furthermore, the DMF-CDs demonstrate excellent biocompatibility and there is no appreciable cytotoxicity observed.

\section{Experimental}

\subsection{Chemicals and materials}

$N, N^{\prime}$-dimethylformamide (DMF), xylene, $N, N^{\prime}$ dimethylacetamide (DMAc), $n$-hexane and cyclohexane were purchased from Sinopharm Chemical Reagent Co. Ltd. (Shanghai, China). Polystyrene (PS) and polymethyl methacrylate (PMMA) were purchased from Aladdin (California, USA). MCF-7 cells were obtained from the Institute of Biochemistry and Cell Biology, Shanghai Institute for Biological Sciences of Chinese Academy of Science (Shanghai, China). Distilled water was used in all the experiment. All the chemicals were used without further purification.

\subsection{Synthesis of the amphiphilic CDs}

Typically, DMF (20 mL) was added to a polyphenol autoclave (50 $\mathrm{mL}$ ) and heated at $260{ }^{\circ} \mathrm{C}$ for 12 hours. After solvothermal reaction, the autoclave was cooled to room temperature and the solution was rotary evaporated to obtain CDs. The distillate was collected to recycle the reagents for the successive runs. The other CDs were synthesized by analogous procedures in which DMF was replaced by DMAc, xylene, $n$-hexane and cyclohexane.

\subsection{Characterization of amphiphilic CDs}

Transmission electron microscopy (TEM) and high-resolution transmission electron microscopy (HRTEM) images were recorded on JEOL JEM-1011 and JEOL JSM-2100, respectively. The CDs were drop-cast onto 400-mesh carbon-coated Cu grids followed by drying in open air at room temperature. The fluorescence spectra of the CDs were recorded on a FluoroMax-4 spectrofluorometer (Horiba Scientific, Japan). X-ray diffraction (XRD) pattern was performed by using a D8 Advance X-ray diffractometer (Bruker) with $\mathrm{Cu} \mathrm{K} \alpha$ radiation. Raman spectra were acquired on a Lab RAM Aramis spectrometer (Horiba Scientific, Japan). Fourier transform infrared (FTIR) spectra were measured on Vector-22 spectrometer (Bruker) ranging from 500 to $4000 \mathrm{~cm}^{-1}$, and the samples were dispersed in $\mathrm{KBr}$ pellets. Ultraviolet-visible (UV-vis) absorption of the CDs solution was obtained using an UV-1800(PC) UV-vis spectrophotometer (Mapada). X-ray photoelectron spectroscopy (XPS) measurements were performed on a PHI 5000 VersaProbe spectrometer (UlVAC-PHI, Japan). The fluorescence confocal images were obtained using a Leica TCS SP5 confocal scanning microscope (Leica Microsystems, Heidelberg $\mathrm{GmbH}$,
Mannheim, Germany). The specimens were excited at $488 \mathrm{~nm}$, and the emission was detected from 500 to $530 \mathrm{~nm}$.

\subsection{Preparation of DMF-CDs-polymer composite}

$0,0.04,0.16$ and $0.2 \mathrm{mg}$ of DMF-CDs were separately added into $5 \mathrm{~mL}$ of DMF together with $1 \mathrm{~g}$ of PMMA or PS. After the polymer was dissolved, the solution was transferred into a watch glass and dried at $60^{\circ} \mathrm{C}$.

\subsection{Cell imaging of DMF-CDs}

MCF-7 cells were incubated in the plates with glucose and $1 \mathrm{~mL}$ Dulbecco's modified Eagle's medium (DMEM) at $37^{\circ} \mathrm{C}$ for $24 \mathrm{~h}$. $0.25 \mathrm{~mL}$ of DMF-CDs aqueous solution $\left(3 \mathrm{mg} \mathrm{mL}^{-1}\right)$ was added into the medium and the final concentration of DMF-CDs were $0.6 \mathrm{mg} \mathrm{mL}{ }^{-1}$. The cells were cultured with the CDs at $37^{\circ} \mathrm{C}$ for $2 \mathrm{~h}$, and washed with the physiological extracellular buffer (ECB: $135 \mathrm{mM} \mathrm{NaCl,} 5 \mathrm{mM} \mathrm{KCl,} 1 \mathrm{mM} \mathrm{CaCl}$, $1 \mathrm{mM} \mathrm{MgCl}_{2}$, $10 \mathrm{mM}$ HEPES, and $10 \mathrm{mM}$ glucose, $\mathrm{pH}$ 7.4) for three times prior to taking the fluorescence image. The fluorescence intensity of the cells was measured using the fluorescence confocal microscope.

\subsection{Cell toxicity of DMF-CDs}

MCF-7 cells $\left(10^{5}\right.$ cells per $\left.\mathrm{mL}\right)$ in DMEM with glucose were cultured in a 96-well microplate $(100 \mu \mathrm{L}$ per well $)$ for $6 \mathrm{~h}$ at $37^{\circ} \mathrm{C}$ with $5 \% \mathrm{CO}_{2}$. Then $100 \mu \mathrm{L}$ of the DMF-CDs solution with different concentrations was introduced into the microplate to acquire the final concentrations of $1,10,100,1000 \mu \mathrm{g} \mathrm{mL}^{-1}$ for the culturing of cells for $24 \mathrm{~h}$. The cells were washed with phosphate-buffered saline (PBS, $\mathrm{pH}$ 7.4). $20 \mu \mathrm{L}$ of $5 \mathrm{mg} \mathrm{mL} \mathrm{m}^{-1}$ MTT and $100 \mu \mathrm{L}$ of DMEM were added to cell wells for $4 \mathrm{~h}$. After removing the culture medium with MTT, $150 \mu \mathrm{L}$ of DMSO was added. The obtained mixture was shaken for $10 \mathrm{~min}$ at room temperature. The optical density (OD) of the mixture was measured at $490 \mathrm{~nm}$ in the En-Spire multimode plate reader. The cell viability was calculated according to the given equation:

$$
\text { Cell viability }=\frac{\mathrm{OD}_{\mathrm{s}}}{\mathrm{OD}_{\mathrm{c}}} \times 100 \%
$$

where $\mathrm{OD}_{\mathrm{s}}$ and $\mathrm{OD}_{\mathrm{c}}$ refer to $\mathrm{OD}$ of samples and control groups, respectively.

\subsection{Quantum yield measurements}

The quantum yield of amphiphilic CDs was measured by the equation below:

$$
\mathrm{QY}_{\mathrm{s}}=\mathrm{QY}_{\mathrm{r}} \frac{I_{\mathrm{s}} A_{\mathrm{r}} \eta_{\mathrm{s}}{ }^{2}}{I_{\mathrm{r}} A_{\mathrm{s}} \eta_{\mathrm{r}}{ }^{2}}
$$

where $\mathrm{s}$ and $\mathrm{r}$ refer to sample and reference. The reference quinine sulfate was dissolved in $0.1 \mathrm{M} \mathrm{H}_{2} \mathrm{SO}_{4}$, of which the quantum yield was 0.54 at $360 \mathrm{~nm} .{ }^{17} \mathrm{I}$ is the integrated emission intensity of fluorescent spectra from 380 to $700 \mathrm{~nm}$ at the excited wavelength of $360 \mathrm{~nm}$. $A$ represents UV-vis absorbance at $360 \mathrm{~nm}$, which is from 0.1 to 0.01 in the $1 \mathrm{~cm}$ quartz 
absorbance cell. $\eta$ refers to the refractive index which is listed in Table $\mathrm{S} 1$ of the ESI $\dagger$.

\subsection{Stability test of DMF-CDs}

$1 \mathrm{~mL}$ of DMF-CDs solution $\left(0.2 \mathrm{mg} \mathrm{mL}^{-1}\right)$ was diluted by $2 \mathrm{~mL}$ of distilled water, which serves as the blank sample. Also $2 \mathrm{~mL}$ of DMF-CDs solution $\left(0.2 \mathrm{mg} \mathrm{mL}^{-1}\right)$ was diluted by $2 \mathrm{~mL} \mathrm{HCl}$ $\left(0.2 \mathrm{mg} \mathrm{mL}^{-1}\right)$, sodium hydroxide solution $\left(0.2 \mathrm{mg} \mathrm{mL}^{-1}\right), 5 \mathrm{mg}$ $\mathrm{mL}^{-1}$ ferric nitrate solution, and $5 \mathrm{mg} \mathrm{mL}{ }^{-1}$ copper sulfate solution, separately. As a result, the five samples were DMF-CDs solution (0.1 $\mathrm{mg} \mathrm{mL}^{-1}$ ) with blank, $\mathrm{HCl}(\mathrm{pH} 1), \mathrm{NaOH}(\mathrm{pH} 13)$, $\mathrm{Fe}\left(\mathrm{NO}_{3}\right)_{3}\left(2.5 \mathrm{mg} \mathrm{mL}{ }^{-1}\right)$, and $\mathrm{Cu}\left(\mathrm{NO}_{3}\right)_{2}\left(2.5 \mathrm{mg} \mathrm{mL}^{-1}\right)$ solution. Then the fluorescent emission spectra of the above samples was tested and compared.

\section{Results and discussion}

Following the procedure depicted in Scheme 1, we prepared a variety of CDs from different organic solvents (Table S2 $\dagger$ ), implying the generality of this method. We speculate that the formation of CDs involves three steps, including decomposition, oxidation/carbonization, and surface passivation. Taking DMF-CDs as a representative example, DMF was decomposed into carbon monoxide and dimethylamine in the first step. ${ }^{\mathbf{1 8}}$ Since the autoclave was airtight and the temperature was high, the decomposition products of DMF molecules was oxidized, carbonized and turned into CDs directly. ${ }^{19}$ Finally, the external surface of CDs was passivated via covalent bonding of aminecontaining agents. ${ }^{20}$ The effect of hydrothermal temperature on the formation of CDs was explored. As shown in Fig. S1 $\uparrow$ (ESI), the lower temperature led to the decrease of fluorescence, while no fluorescence was observed when the reaction temperature was $120{ }^{\circ} \mathrm{C}$. This result indicated that the temperature played a key role in the formation of CDs.

We found that the synthesized DMF-CDs were small and monodisperse, as revealed by transmission electron microscopy (TEM) image in Fig. 1a. No lattice structure was observed in the HRTEM image (Fig. 1b), indicating amorphous dots were formed. Fig. 1c showed the average diameter of the CDs is $3.5 \pm$ $1.0 \mathrm{~nm}$ by measuring 100 particles. The XRD pattern of DMFCDs (Fig. S2, ESI $\dagger$ ) showed a broad diffraction peak at $27.3^{\circ}$, which revealed the amorphous crystal structure of DMF-CDs and conformed to the result of HRTEM. ${ }^{21}$ Raman spectra exhibited weak $\mathrm{D}$ band at around $1314 \mathrm{~cm}^{-1}$ and strong $\mathrm{G}$ band at $1600 \mathrm{~cm}^{-1}$ (Fig. S3, ESI $\dagger$ ), which were related to $\mathrm{sp}^{3}$ carbon and the stretching vibration of $\mathrm{C}=\mathrm{C}$ bonds, respectively. ${ }^{22}$ This

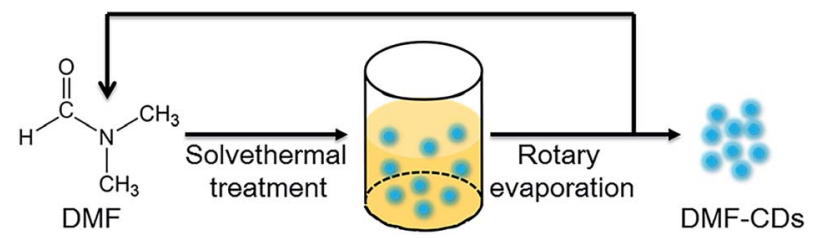

Scheme 1 Schematic illustration of the preparation of DMF-CDs and the cyclic process of DMF and DMF-CDs.

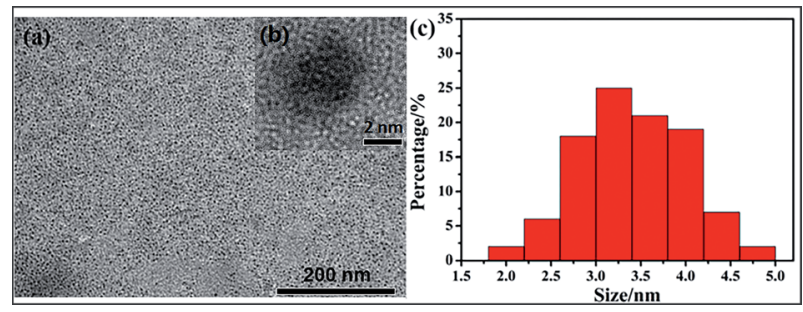

Fig. 1 (a) TEM and (b) HRTEM images of DMF-CDs; (c) size distribution of DMF-CDs.

result indicated the hybrid form of the carbon atoms in DMFCDs was mainly disordered $\mathrm{sp}^{2} .^{23}$

The fluorescence spectra of the solution were measured to investigate the optical property of DMF-CDs. Under ultraviolet (UV) illumination at $365 \mathrm{~nm}$, DMF-CDs showed an intense blue color (Fig. S4b, ESI $\dagger$ ), indicating DMF-CDs had a good fluorescent property. The fluorescence spectra of the solution displayed wavelength-dependent excitation and broad emissions (Fig. S4c, ESI $\dagger$ ). ${ }^{4}$ With the excitation wavelength increasing from 320 to $440 \mathrm{~nm}$, the emission wavelength increased from 420 to $500 \mathrm{~nm}$. Such excitation-dependent photo luminescent behavior was consistent with previously reported CDs, which was probably due to the defect sites from the surface passivation. ${ }^{4 a}$ The quantum yields of the CDs were summarized in Table S2. $\dagger$ The DMF-CDs and DMAc-CDs exhibited the high yields of $33.9 \%$ and $31.8 \%$, which were superior to CDs derived from organic solvents in the previous reports (Table S3, ESI $\dagger$ ). On the contrary, the quantum yields of CDs prepared from other nitrogen-free solvents (i.e., xylene, $n$-hexane and cyclohexane) were much lower $(<13 \%$, Table S2, ESI $\dagger)$. Given the fact that quantum yield of CDs are readily influenced by the composition, ${ }^{24}$ we infer that the intrinsic nitrogen in DMF and DMAc could account for the high quantum yields of DMF-CDs and DMAc-CDs. ${ }^{25}$

In an effort to explore the chemical structure and functional groups on DMF-CDs, UV-vis and FT-IR analyses were performed. The UV-vis absorption spectrum of DMF-CDs had a strong peak centered at $320 \mathrm{~nm}$ (Fig. 2a), typically corresponding to the $\mathrm{n}-\mathrm{p} *$ transition of the $\mathrm{C}=\mathrm{O}$ bond. ${ }^{26}$ The FTIR spectrum of DMF-CDs (Fig. 2b) shows an intense peak at $1600 \mathrm{~cm}^{-1}$, indicating the stretch vibration of carbonyl groups. Additionally, the peaks of other functional groups can be observed in DMF-CDs spectrum, evidenced by their characteristic vibrations centered at $3420 \mathrm{~cm}^{-1}$ (O-H stretching), $2920 \mathrm{~cm}^{-1}$ ( $\mathrm{sp}^{3} \mathrm{C}-\mathrm{H}$ stretch), $1380 \mathrm{~cm}^{-1}\left(\mathrm{sp}^{3} \mathrm{C}-\mathrm{H}\right.$ bending) and $1270 \mathrm{~cm}^{-1}(\mathrm{C}-\mathrm{O}$ stretching). To gain a further insight of the surface composition of CDs, XPS analyses were conducted and the DMF-CDs displayed much lower nitrogen content than DMF in the overall XPS spectra (Fig. 2c). The atomic ratio of N/C in DMF is $1 / 3$ and it became very low in DMF-CDs. The XPS of C1s spectrum (Fig. 2d) was deconvoluted into three bands with the binding energy of 284.6, 286, $286.6 \mathrm{eV}$, corresponding to $\mathrm{C}=\mathrm{C} /$ $\mathrm{C}-\mathrm{C}, \mathrm{C}-\mathrm{N}$, and $\mathrm{C}=\mathrm{O}$, respectively. The N1s spectrum (Fig. S5a, ESI $\dagger$ ) exhibited two peaks at 400.2 and $398.6 \mathrm{eV}$, which were assigned to $\mathrm{N}-\mathrm{H}$ and $\mathrm{C}-\mathrm{N}-\mathrm{C}$ groups, respectively. For the $\mathrm{O} 1 \mathrm{~s}$ 

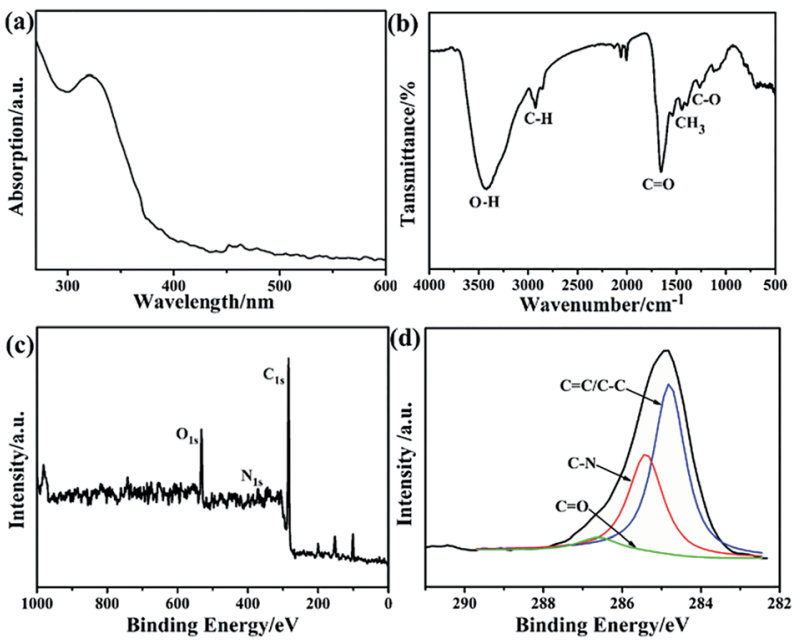

Fig. 2 (a) UV-vis absorption and (b) FT-IR spectra of DMF-CDs; (c) XPS survey of DMF-CDs; (d) XPS fitting of C1s in DMF-CDs.

spectrum of DMF-CDs (Fig. S5b, ESI $\dagger$ ), the two peaks at 530.4 and $529.6 \mathrm{eV}$ were attributed to $\mathrm{C}-\mathrm{OH} / \mathrm{C}-\mathrm{O}-\mathrm{C}$ and $\mathrm{C}=\mathrm{O}$, respectively. On the basis of the combined results from UV-vis, FTIR and XPS measurements, the as-prepared DMF-CDs have various functional groups such as $\mathrm{N}-\mathrm{H}, \mathrm{C}-\mathrm{O}-\mathrm{C}, \mathrm{C}=\mathrm{O}$ on the external surface due to the surface passivation with DMF during the synthesis. These functional groups impart good amphiphilic properties of DMF-CDs without further chemical modification.

In order to investigate the chemical stability and the effect of metal ions on the emission of the DMF-CDs, we measured their fluorescence under different $\mathrm{pH}$ or in the presence of $\mathrm{Fe}^{3+} / \mathrm{Cu}^{2+}$ ions. As shown in Fig. 3, there is no significant loss (less than $5 \%$ ) in the fluorescence intensity of DMF-CDs. The result showed that the DMF-CDs possessed high ion and pH stability and could emit stable fluorescence in different solutions.

Since the DMF-CDs were synthesized in/from DMF, the DMFCDs are readily soluble in DMF. Considering DMF is a good solvent for dissolving polymers, we resort to dope CDs into polymers, such as polymethylmethacrylate (PMMA) and polystyrene (PS), with the aim to explore the potential of using amphiphilic CDs as fluorescent inks. As shown in Fig. 4, PS and

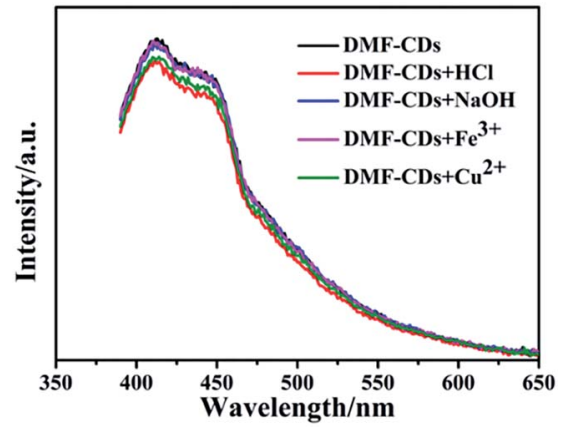

Fig. 3 Emission spectra (excited at $360 \mathrm{~nm}$ ) of DMF-CDs in hydrochloric acid, sodium hydroxide, ferric nitrate and copper sulphate solutions. (a)

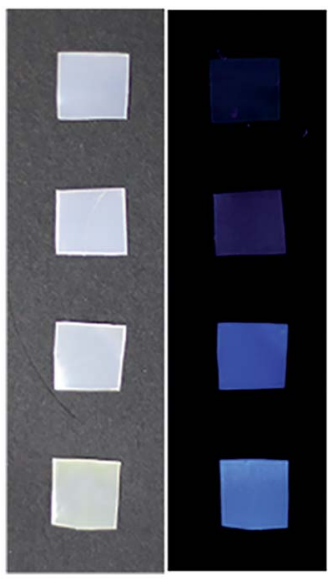

(b)

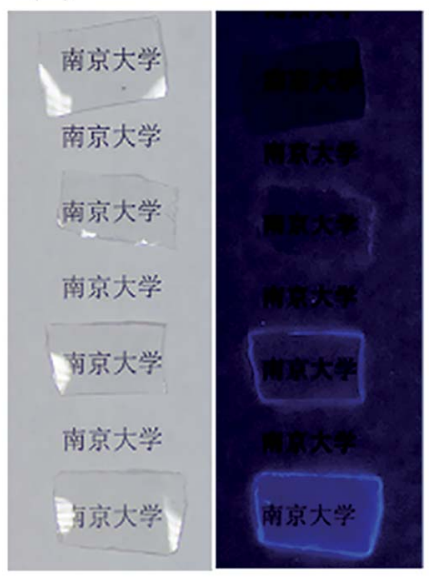

Fig. 4 Photographs of (a) PMMA and (b) PS films doped with DMF-CD sunder white light (left) and UV light (right, $365 \mathrm{~nm}$ ). DMF-CDs mass concentration in the PMMA and PS films (top-down): 0, 0.04, 0.08, and $0.2 \%$

PMMA films emit blue light after loading CDs. Increasing the concentration of DMF-CDs lead to enhanced intensity of emitting light from the polymers. No fluorescence light was observed from the bare polymers, indicating the DMF-CDs are the only fluorescence source. Impressively, these CDs-polymer composites are highly stable and emitted blue fluorescent light without obvious decay even after one year. The addition of hydrochloric acid, sodium hydroxide, ferric nitrate and copper sulfate solution, had negligible effect on the intensity of fluorescence. On the basis of the above results, the DMF-CDspolymer composites were quite stable in different solutions. All these results uncover the important potential of DMF-CDs as promising ink for printing.

By virtue of their strong fluorescence and tunable excitation and emission wavelength, CDs emerged as promising candidates in optical bio-imaging. We sought to evaluate the cytotoxicity of the DMF-CDs, which have the highest quantum yield in the present study. The DMF-CDs were dispersed into water with ultrasonic treatment for $10 \mathrm{~min}$ to obtain the aqueous CD solution. Notably, the photoluminescence emission spectra and particle size of DMF-CDs did not show obvious changes in $\mathrm{H}_{2} \mathrm{O}$ compared to that in DMF (Fig. S6 and S7, ESI $\dagger$ ). DMF-CDs were introduced into the cells and the cells emitted green light upon $490 \mathrm{~nm}$ excitation (Fig. 5). The observation of fluorescence at cells confirmed that the DMF-CDs were water-soluble, and could be uptaken into the cells. The cytotoxicity of the carbon dots was tested (Fig. S8, ESI $\dagger$ ) and the DMF-CDs rendered extremely low toxicity below $100 \mu \mathrm{g} \mathrm{mL}{ }^{-1}$. In addition, there was no obvious morphological cell change observed after incubation with the CDs, further demonstrating their prominent biocompatibility. The abovementioned results unambiguously imply that the DMF-CDs, featuring low toxicity and high quantum yield, hold great potential in biological applications, such as bio-imaging and protein analysis by deposit. 


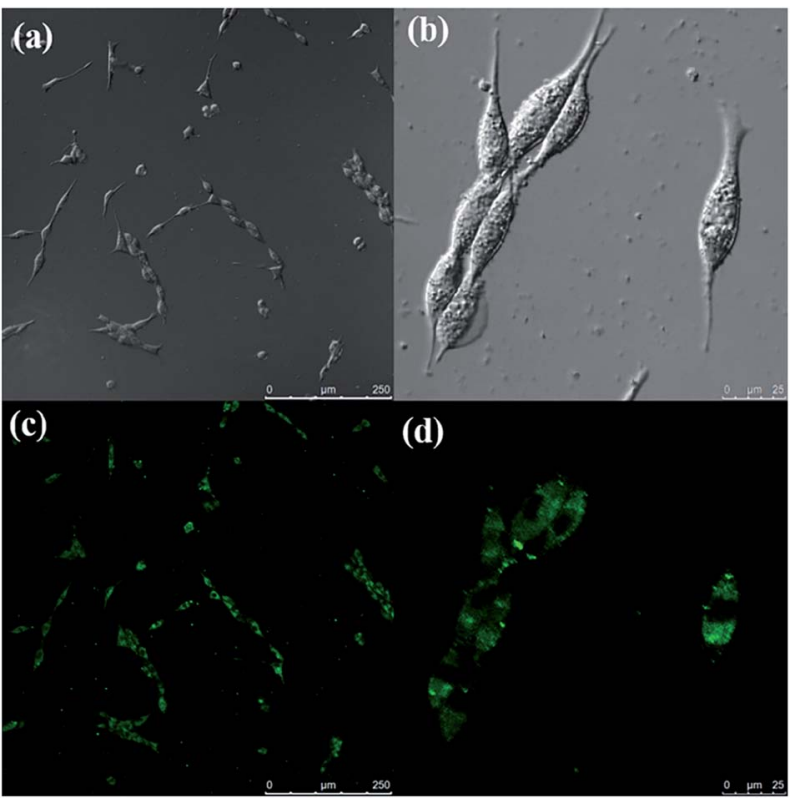

Fig. 5 Representative ( $a$ and b) bright field and ( $c$ and d) fluorescence microphotographs of MCF-7 cells labelled with DMF-CDs.

\section{Conclusions}

In conclusion, we have successfully prepared a series of CDs via a green one-step hydrothermal synthesis route without any catalyst, additives or by-products in a recycle manner. Among the CDs series, DMF- and DMAc-CDs possess amphiphilic feature and enhanced quantum yields, presumably due to the presence of nitrogen. The prepared DMF-CDs have small size with a narrow size distribution. Moreover, the oxygen/nitrogen doped structure derived from simultaneous surface passivation endows them with strong and excitation-dependent fluorescence. Due to the amphiphilic feature, remarkable luminescence stability, and good biocompatibility, DMF-CDs could be readily doped into the polymers and the robust CDspolymers composites exhibited high fluorescence. Additionally, the DMF-CDs were appropriately uptaken by cells with low cytotoxicity, thus demonstrating their potential for fluorescent cellular imaging. This green method opens up fascinating opportunities for the large-scale synthesis of CDs with the immense potential in fluorescent composites synthesis and biological imaging.

\section{Conflicts of interest}

There are no conflicts of interest to declare.

\section{Acknowledgements}

This study was supported by the Fundamental Research Funds for the Central Universities (No. 1104020505, 020514380111) and the Postgraduate Research and Innovation Project of Jiangsu Province (2014, No. KYLX_0030).

\section{Notes and references}

1 V. Strauss, J. T. Margraf, C. Dolle, B. Butz, T. J. Nacken, J. Walter, W. Bauer, W. Peukert, E. Spiecker, T. Clark and D. M. Guldi, J. Am. Chem. Soc., 2014, 136, 17308.

2 G. Eda, Y.-Y. Lin, C. Mattevi, H. Yamaguchi, H.-A. Chen, I. S. Chen, C.-W. Chen and M. Chhowalla, Adv. Mater., 2010, 22, 505.

3 J. Shen, Y. Zhu, C. Chen, X. Yang and C. Li, Chem. Commun., 2011, 47, 2580.

4 (a) Y.-P. Sun, B. Zhou, Y. Lin, W. Wang, K. A. S. Fernando, P. Pathak, M. J. Meziani, B. A. Harruff, X. Wang, H. Wang, P. G. Luo, H. Yang, M. E. Kose, B. Chen, L. M. Veca and S.-Y. Xie, J. Am. Chem. Soc., 2006, 128, 7756; (b) Y. Song, S. Zhu and B. Yang, RSC Adv., 2014, 4, 27184.

5 A. P. Alivisatos, Science, 1996, 271, 933.

6 (a) D. Hazarika and N. Karak, Green Chem., 2016, 18, 5200; (b) J. Gu, W. Wang, Q. Zhang, Z. Meng, X. Jia and K. Xi, RSC Adv., 2013, 3, 15589.

7 J. Gu, D. Hu, W. Wang, Q. Zhang, M. Zhen, X. Jia and X. Kai, Biosens. Bioelectron., 2015, 68, 27.

8 A. M. Craciun, A. Diac, M. Focsan, C. Socaci, K. Magyari, D. Maniu, I. Mihalache, L. M. Veca, S. Astilean and A. Terec, $R S C A d v$. , 2016, 6, 56944.

9 (a) X. Zhou, S. Guo, P. Zhong, Y. Xie, Z. Li and X. Ma, RSC Adv., 2016, 6, 54644; (b) A. A. Ensafi, S. H. Sefat, N. Kazemifard, B. Rezaei and F. Moradi, Sens. Actuators, B, 2017, 253, 451.

10 H. Zhu, X. Wang, Y. Li, Z. Wang, F. Yang and X. Yang, Chem. Commun., 2009, 5118.

11 S.-L. Hu, K.-Y. Niu, J. Sun, J. Yang, N.-Q. Zhao and X.-W. Du, J. Mater. Chem., 2009, 19, 484.

12 (a) Z.-C. Yang, M. Wang, A. M. Yong, S. Y. Wong, X.-H. Zhang, H. Tan, A. Y. Chang, X. Li and J. Wang, Chem. Commun., 2011, 47, 11615; (b) S. Sahu, B. Behera, T. K. Maiti and S. Mohapatra, Chem. Commun., 2012, 48, 8835; (c) Y. Yang, J. Cui, M. Zheng, C. Hu, S. Tan, Y. Xiao, Q. Yang and Y. Liu, Chem. Commun., 2012, 48, 380; (d) M. Zhou, Z. Zhou, A. Gong, Y. Zhang and Q. Li, Talanta, 2015, 143, 107.

13 B. Kong, A. Zhu, C. Ding, X. Zhao, B. Li and T. Yang, Adv. Mater., 2012, 24, 5844.

14 (a) Y. Wang, W. Qi and Y. Song, Chem. Commun., 2016, 52, 7994; (b) M. Li, F. Xu, H. Li and Y. Wang, Catal. Sci. Technol., 2016, 6, 3670; (c) Q. Lu, J. Deng, Y. Hou, H. Wang, H. Li, Y. Zhang and S. Yao, Chem. Commun., 2015, 51, 7164.

15 (a) Z. Yang, X. Zheng, Z. Li and J. Zheng, Analyst, 2016, 141, 4757; (b) G. Li, N. Lv, W. Bi, J. Zhang and J. Nia, New J. Chem., 2016, 40, 10213.

16 M. Zheng, S. Liu, J. Li, D. Qu, H. Zhao, X. Guan, X. Hu, Z. Xie, X. Jing and Z. Sun, Adv. Mater., 2014, 26, 3554.

17 W. H. Melhuish, J. Phys. Chem., 1961, 65, 229.

18 (a) J. Muzart, Tetrahedron, 2009, 65, 8313; (b) S. Liu, J. Tian, L. Wang, Y. Zhang, Y. Luo, A. M. Asiri, A. O. Al-Youbi and X. Sun, RSC Adv., 2012, 2, 4632. 
19 (a) J. Deng, Q. Lu, N. Mi, H. Li, M. Liu, M. Xu, L. Tan, Q. Xie, Y. Zhang and S. Yao, Chem.-Eur. J., 2014, 20, 4993; (b) J. Gu, D. Hu, J. Huang, X. Huang, Q. Zhang, X. Jia and K. Xi, Nanoscale, 2016, 8, 3973.

20 (a) L. Sai, J. Chen, Q. Chang, W. Shi, Q. Chen and L. Huang, RSC Adv., 2017, 7, 16608; (b) J. Chen, Q. Wang, J. Zhou, W. Deng, Q. Yu, X. Cao, J. Wang, F. Shao, Y. Li, P. Ma, M. Spector, J. Yu and X. Xu, Nanoscale, 2017, 9, 10820.

21 B. Fang, P. Wang, Y. Zhu, C. Wang, G. Zhang, X. Zheng, C. Ding, J. Gu and F. Cao, RSC Adv., 2018, 8, 7377.
22 V. B. Kumar, A. K. Sahu, A. S. M. Mohsin, X. Li and A. Gedanken, ACS Appl. Mater. Interfaces, 2017, 9, 28930.

23 W. Zhu, J. Zhang, Z. Jiang, W. Wang and X. Liu, RSC Adv., 2014, 4, 17387.

24 (a) Y. Dong, H. Pang, H. B. Yang, C. Guo, J. Shao, Y. Chi, C. M. Li and T. Yu, Angew. Chem., Int. Ed., 2013, 52, 7800; (b) X. Zhai, P. Zhang, C. Liu, T. Bai, W. Li, L. Dai and W. Liu, Chem. Commun., 2012, 48, 7955.

25 J. Hou, W. Wang, T. Zhou, B. Wang, H. Li and L. Ding, Nanoscale, 2016, 8, 11185.

26 C. Shen, Y. Sun, J. Wang and Y. Lu, Nanoscale, 2014, 6, 9139. 